Journal of Research in Interprofessional

Practice and

Education

Vol. 6.1

April 2016
Journal of Research in Interprofessional Practice and Education (JRIPE)

Vol. 6.1

(C) 2016

Corresponding author: Catherine L. Hatfield.

Email: chatfield@uh.edu

\section{Changes in Pharmacy Students After Experiencing Interprofessional Education Activities}

\author{
Shane R. Tolleson, PharmD; Nadia J. Ismail, MD, MPH, MEd, FACP; \\ Anne C. Gill, DrPH, MS; Rita A. Dello Stritto, PhD, RN, CNS, ENP, \\ ACNP-BC; Mark D. Hatfield, MS, PhD Candidate; Cayla R. Teal, PhD; \\ Elizabeth A. Nelson, MD; \& Catherine L. Hatfield, PharmD
}

\begin{abstract}
Background: Interprofessional education (IPE) has become increasingly important among healthcare professionals. The purpose of this study was to assess the impact of IPE experiences on pharmacy students' perceived level of comfort with and level of reliability regarding other healthcare professionals.

Methods and Findings: A survey of third- and fourth-year pharmacy students $(N=190)$ was analyzed using descriptive statistics and bivariate analyses to address the study objectives. Students experienced a significant increase in their level of comfort with questioning other professions after IPE (pre IPE: $5.45 \pm 2.24$ and post IPE: $7.22 \pm 1.62$, $p<0.01$ ) and being questioned by other professions (pre IPE: $5.39 \pm 2.24$ and post IPE: $7.04 \pm 1.68, p<0.01$ ). Students also experienced a significant increase in the perceived reliability and accuracy of information from nurses after IPE (pre IPE: $5.91 \pm 1.82$ and post IPE: $6.41 \pm 1.62, p=0.01$ ).

Conclusions: Pharmacy students who experienced any IPE perceived themselves as more comfortable with questioning and being questioned by other healthcare professionals than students without IPE. Additional research is needed to more clearly define the benefits of IPE and how it should be designed.
\end{abstract}

Keywords: Interprofessional education; IPE; Pharmacy student; Behaviour; Attitude

\section{Introduction}

With healthcare becoming more complex and with more emphasis placed on improved outcomes and safety, interprofessional collaboration among healthcare professionals is gaining more importance. The increased significance placed upon interprofessional collaboration has led to changes in the education of healthcare professionals, with greater focus aimed at developing interprofessional education (IPE) by accrediting bodies, such as the American Association of Colleges of Pharmacy (AACP) [1]. The Centre for the Advancement of Interprofessional Education defines 
2

Changes in

Pharmacy Students After Experiencing Interprofessional Education Activities

Tolleson, Ismail, Gill, Dello Stritto, Hatfield, Teal, Nelson, \& Hatfield
Journal of Research in Interprofessional Practice and Education

Vol. 6.1

April 2016
IPE as occurring when two or more professions learn with, from, and about each other to improve collaboration and the quality of care [2]. Implementation of IPE is useful for developing the collaborative skills needed by healthcare professionals to work as members of interprofessional teams, in which each member has the common goal of improving patient care [3].

Healthcare students who experience IPE in their curriculum are exposed to situations that allow them to better understand their roles, other members' roles, and team values when caring for patients [3]. IPE experiences should focus on students learning to collaborate seamlessly to improve patient care, while using their different knowledge bases and training from different areas of healthcare [4]. Students are able to take their IPE experience and apply it to future practice with the purpose of improving patient care [1]. These improvements stem from students learning important skills from IPE, such as communication, reflection, and acceptance of other opinions, and then applying those skills to their work [5]. Interprofessional experiences are prime opportunities for healthcare professionals to develop themselves and prepare for the current healthcare field, while still in a controlled and constructive environment.

The current AACP Accreditation Standards and Guidelines mention IPE as a focus, and list it in several of the standards and guidelines [6]. In 2009, AACP joined five other national health profession education associations to form the Interprofessional Education Collaborative (IPEC), with the purpose of helping prepare future health professionals for interprofessional patient care teams. Despite the emphasis on IPEs, many pharmacy schools still do not have IPE as part of their curriculum. In 2011, a survey conducted by faculty members from pharmacy schools in the US found that 45 percent (41 out of 91) of pharmacy schools that responded did not currently have IPE integrated into their Introductory Pharmacy Practice Experiences (IPPEs) [7].

Currently, limited data is available to support the belief that IPE translates to changes in healthcare practice [8]. Research regarding IPE has been conducted since the late 1970s, however, the conditions of the IPE experiences have been highly variable, making them difficult to compare and generalize $[1,8]$. Fortunately, literature regarding pharmacy students experiencing IPE is beginning to become available [9-11]. The primary objective of this study was to assess the impact of IPE experiences on pharmacy students' perceived level of comfort with and level of reliability regarding other healthcare professionals. The secondary objective was to investigate the differences in baseline perceptions among pharmacy students who experienced IPE versus students who did not.

\section{Methods}

\section{Study design}

Pharmacy students from the University of Houston College of Pharmacy (UHCOP) enrolled in the fall semester of 2013 as third-year (P3) or fourth-year (P4) students were selected to participate in this study. These students were chosen because they were given opportunities to participate in IPE activities as third-year students 
3

Changes in

Pharmacy Students After Experiencing Interprofessional Education Activities

Tolleson, Ismail, Gill, Dello Stritto, Hatfield, Teal, Nelson, \& Hatfield

Journal of Research in Interprofessional Practice and Education

Vol. 6.1

April 2016 through the optional No Place Like Home (NPLH) Clinical Visit, which was part of the Longitudinal Ambulatory Clinical Experience course (LACE course) at Baylor College of Medicine (BCM), and/or a required interprofessional simulation experience [12]. Three subgroups of IPE students (who experienced only the LACE course, those who experienced only the P4 IPE, and those who experienced both) were compared with each other and with students who had no IPE.

The LACE course NPLH activity is an interprofessional education activity involving medical students from BCM and pharmacy students from UHCOP. During this activity, two medical students and two pharmacy students are grouped with a geriatric practitioner. The group travels together to make house calls to two patients who are unable to leave their home for a clinic visit. The students assess the patients with the practitioner and address the patients' problems. The LACE course is an optional IPE for third-year pharmacy students because there are not enough slots to require the entire class to participate.

The required IPE for fourth-year students is a simulation exercise in which medical students from BCM, nursing students from Texas Woman's University (TWU), and pharmacy students from UHCOP work as a team in a simulated patient-care experience. The teams are comprised of one medical student, one nursing student, and one pharmacy student. Each team must work together to communicate a medication error to a simulated patient family member. The IPE scenario was developed to create a shared error in which the physician wrote an inappropriate dose for a drug order, the pharmacist dispensed the order, and the nurse administered the medication. The IPE begins with the team getting to know each other. The students are then provided information about their roles in a medication error and have to prepare as a team to meet an emotional family member to divulge the error. After meeting with the family member, the team moves into a debriefing session led by educators from each discipline. The goals of this IPE are to recognize the individual expertise each profession brings to patient care delivery, foster respect and trust for members of an interprofessional working team, and broaden the scope of effective communication about challenging interprofessional team issues.

\section{Data collection}

IPE faculty and a fourth-year pharmacy student at UHCOP developed a 38-item instrument that faculty involved in IPEs in the study's pharmacy, nursing, and medical schools determined to have face validity. The instrument was administered online to pharmacy students using Qualtrics ${ }^{\circledR}$ software (developed by Qualtrics in Provo, UT). The instrument incorporated a retrospective pre-test and post-test to capture pharmacy students' perceived level of comfort with and level of reliability regarding other healthcare professionals before and after experiencing an IPE activity. The retrospective pre-test method was used to minimize possible "response shift bias," which could result using a traditional pre-test and post-test survey, thus threatening internal validity [13]. Response shift refers to the recalibration of a person's basis for a particular dimension as a result of a deeper understanding of the dimension after experiencing it first-hand [14]. 
Changes in Pharmacy Students After Experiencing Interprofessional Education Activities

Tolleson, Ismail, Gill, Dello Stritto, Hatfield, Teal, Nelson, \& Hatfield

Journal of Research in Interprofessional Practice and Education

Vol. 6.1

April 2016
Journal of Research in Interprofessional Practice and Education

At the time the survey was opened, approximately half of the fourth-year pharmacy students (P4s) had already completed their required IPE activity. Additionally, approximately one third of the P3 students and half of the P4 students experienced the LACE NPLH visit. The timing of the survey was designed to gather fairly balanced data from both groups of students. The survey was anonymous, with no identifiers recorded. The survey collected demographic information, including gender, year in pharmacy school, age, current attitudes toward the medical and nursing professions, and if students had any personal connection to the medical or nursing professions. The last question in the demographics section was used to sort students according to whether they had already taken part in IPE activities or not. Based on this response, students who previously participated in IPE (as a P3 and/or a P4) were directed to questions aimed to detect a change in perceived behaviour related to practice and changes in perceived attitude toward the medical and nursing professions. Students who had not previously participated in IPE were directed to questions designed to measure perceived attitudes toward the other professions.

To address the primary objective, four sliding scale questions were common to all students. The first question assessed the perceived level of comfort with questioning a member of another health profession. The second question assessed the perceived level of comfort with a member of another health profession questioning the pharmacy student. These responses were recorded on a sliding scale from 0 being the least comfortable to 10 being the most comfortable. The third question assessed the perceived level of reliability or accuracy of information from a nurse or nursing student. The fourth question assessed the perceived level of reliability or accuracy of information from a physician or medical student. These responses were recorded on a sliding scale from 0 being the least reliable to 10 being the most reliable. A copy of the questionnaire is included in the Appendix.

A link to the survey was emailed to each student. Reminder emails were sent and word of mouth was used to remind the students to complete the survey. A total of 222 students were included in the emails (107 fourth-year and 115 third-year students). The survey was closed after 10 days. The University of Houston Institutional Review Board approved this research.

\section{Statistical analysis}

Data collected by Qualtrics ${ }^{\circledR}$ was exported to an Excel ${ }^{\circledR}$ (Microsoft Corp., Redmond, WA) spreadsheet and subsequently exported to SAS ${ }^{\circledR}$ version 9.3 (SAS Institute Inc., Cary, NC) for descriptive statistics and statistical analysis. Chi-square tests or Fisher's exact tests were performed on all categorical variables where appropriate. Independent $t$-tests were conducted for comparisons of means across subjects, and paired $t$-tests were conducted for comparisons within subjects. The level of significance was set at an a priori level of $p \leq 0.05$.

\section{Results}

The survey response rate was 85.6 percent (190/222). Most were female (62.1\%) and $26.2 \pm 4.1$ years old, which is consistent with the class demographics. Additionally, 
JRIPE

5

Changes in

Pharmacy Students After Experiencing Interprofessional Education Activities

Tolleson, Ismail, Gill, Dello Stritto, Hatfield, Teal, Nelson, \& Hatfield

the responses were evenly divided between P3 or P4 years (49.5\% and 50.5\%, respectively). See Table 1 for demographic results.

Table 1. Demographic description of sample $(n=190)$

\begin{tabular}{|c|c|c|c|c|c|c|}
\hline Item & Category & No IPE & P3 LACE & P4 IPE & Any IPE & Total \\
\hline $\begin{array}{l}\text { Gender, } \\
n(\%)^{\mathrm{a}}\end{array}$ & $\begin{array}{l}\text { Male } \\
\text { Female }\end{array}$ & $\begin{array}{l}43(41.8) \\
60(58.3)\end{array}$ & $\begin{array}{c}6(24.0) \\
19(76.0)\end{array}$ & $\begin{array}{l}18(54.5) \\
15(45.5)\end{array}$ & $\begin{array}{c}5(17.2) \\
24(82.8)\end{array}$ & $\begin{array}{c}72(37.9) \\
118(62.1)\end{array}$ \\
\hline $\begin{array}{l}\text { Year, } \\
n(\%)^{\mathrm{a}}\end{array}$ & $\begin{array}{l}\text { P3 } \\
\text { P4 }\end{array}$ & $\begin{array}{l}81(78.6) \\
22(21.4)\end{array}$ & $\begin{array}{l}12(48.0) \\
13(52.0)\end{array}$ & $\begin{array}{c}0(0) \\
33(100.0)\end{array}$ & $\begin{array}{c}1(3.5) \\
28(96.6)\end{array}$ & $\begin{array}{l}94(49.5) \\
96(50.5)\end{array}$ \\
\hline $\begin{array}{l}\text { Age, Mean } \pm \text { SD } \\
(n)\end{array}$ & & $\begin{array}{c}26.1 \pm 4.1 \\
(103)\end{array}$ & $\begin{array}{l}26.8 \pm 4.7 \\
(25)\end{array}$ & $\begin{array}{c}25.6 \pm 2.9 \\
(33)\end{array}$ & $\begin{array}{c}26.7 \pm 4.9 \\
(29)\end{array}$ & $\begin{array}{c}26.2 \pm 4.1 \\
(190)\end{array}$ \\
\hline
\end{tabular}

Note: a Percentages may not sum to $100.0 \%$ due to rounding.

Table 2 compares characteristics of responses between students with no IPE experience and students with any IPE experience. The only statistically significant finding was that IPE students expressed only positive or neutral attitudes toward the medical profession versus non-IPE students who had more neutral and some negative attitudes. The other items were relatively balanced across these two groups: previously worked with nurses, nursing students, physicians, or medical students; know any nurses, nursing students, physicians, or medical students; and attitude toward the nursing profession.

Table 2. Descriptive characteristics for all respondents $(n=190)$

\begin{tabular}{|c|c|c|c|}
\hline Item & Response & $\begin{array}{l}\text { No IPE, } \\
\text { N (\%) }\end{array}$ & $\begin{array}{c}\text { Any IPE, } \\
\mathrm{N}(\%)^{\mathrm{a}}\end{array}$ \\
\hline Previously worked with nurses or nursing students ${ }^{b}$ & $\begin{array}{l}\text { Yes } \\
\text { No }\end{array}$ & $\begin{array}{l}33(32.0) \\
70(68.0)\end{array}$ & $\begin{array}{l}39(44.8) \\
48(55.2)\end{array}$ \\
\hline Know any nurses or nursing students ${ }^{b}$ & $\begin{array}{l}\text { Yes } \\
\text { No }\end{array}$ & $\begin{array}{l}87(84.5) \\
16(15.5)\end{array}$ & $\begin{array}{l}65(74.7) \\
22(25.3)\end{array}$ \\
\hline Attitude towards nursing profession ${ }^{\mathrm{b}}$ & $\begin{array}{l}\text { Positive } \\
\text { Neutral } \\
\text { Negative }\end{array}$ & $\begin{array}{c}77(74.8) \\
16(15.5) \\
10(9.7)\end{array}$ & $\begin{array}{c}73(83.9) \\
12(13.8) \\
2(2.3)\end{array}$ \\
\hline Previously worked with physicians or medical students ${ }^{b}$ & $\begin{array}{l}\text { Yes } \\
\text { No }\end{array}$ & $\begin{array}{l}66(64.1) \\
37(35.9)\end{array}$ & $\begin{array}{l}57(65.5) \\
30(34.5)\end{array}$ \\
\hline Know any physicians or medical students ${ }^{b}$ & $\begin{array}{l}\text { Yes } \\
\text { No }\end{array}$ & $\begin{array}{l}86(83.5) \\
17(16.5)\end{array}$ & $\begin{array}{l}73(83.9) \\
14(16.1)\end{array}$ \\
\hline Attitude towards medical professionc & $\begin{array}{l}\text { Positive } \\
\text { Neutral } \\
\text { Negative }\end{array}$ & $\begin{array}{c}73(70.9) \\
21(20.4) \\
9(8.7)\end{array}$ & $\begin{array}{c}73(83.9) \\
14(16.1) \\
0(0)\end{array}$ \\
\hline
\end{tabular}

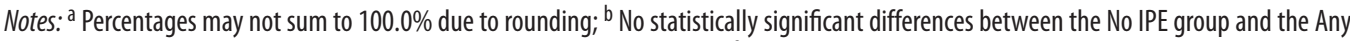
IPE group using either Chi-square tests or Fisher's exact test, as appropriate; ${ }^{C} p=0.01$. Statistically significant difference between the No IPE group and the Any IPE group using Chi-square test.

Journal of Research in Interprofessional

Practice and

Education

Vol. 6.1

April 2016 
JRIPE

6

Changes in

Pharmacy Students After Experiencing Interprofessional

Education Activities

Tolleson, Ismail, Gill, Dello Stritto, Hatfield, Teal, Nelson, \& Hatfield

Table 3 compares the responses across the three different levels of IPE experience (LACE as P3, IPE as P4, and LACE as P3 and IPE as P4 combined). No statistically significant difference was found between the following items: IPE was beneficial (94.3\% agreed overall); previous positive or negative experiences with nurses, nursing students, physicians, or medical students; IPE experience affected how you work with other professions (80.5\% agreed); perception of other professions changed after IPE (62.4\% agreed); more willing to ask for assistance, clarification, or services of physicians or nurses (77.7\% agreed); IPE affected work interaction with physicians or nurses (100\% agreed); and how has the perception of other professions changed (96.2\% responded "better" versus "worse").

Table 3. Responses to questions for students who have participated in interprofessional education (IPE) activities

\begin{tabular}{|c|c|c|c|c|c|}
\hline Item & Response & $\begin{array}{l}\text { P3 LACE, } \\
n(\%)^{\mathrm{a}}\end{array}$ & $\begin{array}{l}\text { P4 IPE, } \\
n(\%)^{\mathrm{d}}\end{array}$ & $\begin{array}{l}\text { P3 LACE \& } \\
\text { P4 IPE, } \\
n(\%)^{\mathrm{a}}\end{array}$ & $\begin{array}{c}\text { Total for P3 LACE } \\
\text { and/or P4 IPE, } \\
n(\%)^{\mathrm{a}}\end{array}$ \\
\hline $\begin{array}{l}\text { Overall, the IPE you participated } \\
\text { in was beneficial }\end{array}$ & $\begin{array}{l}\text { Yes } \\
\text { No }\end{array}$ & $\begin{array}{c}24(96.0) \\
1(4.0)\end{array}$ & $\begin{array}{r}29(87.9) \\
4(12.1)\end{array}$ & $\begin{array}{c}29(100.0) \\
0(0)\end{array}$ & $\begin{array}{c}82(94.3) \\
5(5.8)\end{array}$ \\
\hline $\begin{array}{l}\text { Previous positive experience with } \\
\text { nurses or nursing students }{ }^{\mathrm{b}}\end{array}$ & $\begin{array}{l}\text { Yes } \\
\text { No }\end{array}$ & $\begin{array}{l}19(76.0) \\
6(24.0)\end{array}$ & $\begin{array}{l}25(75.8) \\
8(24.2)\end{array}$ & $\begin{array}{c}26(89.7) \\
3(10.3)\end{array}$ & $\begin{array}{l}70(80.5) \\
17(19.5)\end{array}$ \\
\hline $\begin{array}{l}\text { Previous negative experience with } \\
\text { nurses or nursing students }\end{array}$ & $\begin{array}{l}\text { Yes } \\
\text { No }\end{array}$ & $\begin{array}{c}9(36.0) \\
16(64.0)\end{array}$ & $\begin{array}{l}18(54.6) \\
15(45.5)\end{array}$ & $\begin{array}{l}15(51.7) \\
14(48.3)\end{array}$ & $\begin{array}{l}42(48.3) \\
45(51.7)\end{array}$ \\
\hline $\begin{array}{l}\text { Previous positive experience with } \\
\text { physicians or medical students }{ }^{b}\end{array}$ & $\begin{array}{l}\text { Yes } \\
\text { No }\end{array}$ & $\begin{array}{r}22(88.0) \\
3(12.0)\end{array}$ & $\begin{array}{c}33(100.0) \\
0(0)\end{array}$ & $\begin{array}{l}25(86.2) \\
4(13.8)\end{array}$ & $\begin{array}{c}80(92.0) \\
7(8.1)\end{array}$ \\
\hline $\begin{array}{l}\text { Previous negative experience with } \\
\text { physicians or medical students }{ }^{b}\end{array}$ & $\begin{array}{l}\text { Yes } \\
\text { No }\end{array}$ & $\begin{array}{l}13(52.0) \\
12(48.0)\end{array}$ & $\begin{array}{l}21(63.6) \\
12(36.4)\end{array}$ & $\begin{array}{l}16(55.2) \\
13(44.8)\end{array}$ & $\begin{array}{l}50(57.5) \\
37(42.5)\end{array}$ \\
\hline $\begin{array}{l}\text { IPE affected how you work with } \\
\text { other professions }{ }^{b}\end{array}$ & $\begin{array}{l}\text { Yes } \\
\text { Neutral } \\
\text { No }\end{array}$ & $\begin{array}{l}19(76.0) \\
0(0) \\
6(24.0)\end{array}$ & $\begin{array}{l}25(75.8) \\
0(0) \\
8(24.2)\end{array}$ & $\begin{array}{c}26(89.7) \\
0(0) \\
3(10.3)\end{array}$ & $\begin{array}{c}70(80.5) \\
0(0) \\
17(19.5)\end{array}$ \\
\hline $\begin{array}{l}\text { Perception of other professions } \\
\text { changed after IPE }\end{array}$ & $\begin{array}{l}\text { Yes } \\
\text { No }\end{array}$ & $\begin{array}{l}17(70.8) \\
7(29.2)\end{array}$ & $\begin{array}{l}19(59.4) \\
13(40.6)\end{array}$ & $\begin{array}{l}17(58.6) \\
12(41.4)\end{array}$ & $\begin{array}{l}53(62.4) \\
32(37.7)\end{array}$ \\
\hline $\begin{array}{l}\text { More willing to ask for assistance, } \\
\text { clarification, or services of physi- } \\
\text { cians or nurses }\end{array}$ & $\begin{array}{l}\text { Yes } \\
\text { Neutral } \\
\text { No }\end{array}$ & $\begin{array}{l}21(87.5) \\
2(8.3) \\
1(4.2)\end{array}$ & $\begin{array}{c}20(62.5) \\
10(31.3) \\
2(6.3)\end{array}$ & $\begin{array}{c}25(86.2) \\
4(13.8) \\
0(0)\end{array}$ & $\begin{array}{l}66(77.7) \\
16(18.8) \\
3(3.5)\end{array}$ \\
\hline $\begin{array}{l}\text { IPE affected work interaction with } \\
\text { physicians or nurses }{ }^{b}\end{array}$ & $\begin{array}{l}\text { Positively } \\
\text { Negatively }\end{array}$ & $\begin{array}{c}19(100.0) \\
0(0)\end{array}$ & $\begin{array}{c}16(100.0) \\
0(0)\end{array}$ & $\begin{array}{c}20(100.0) \\
0(0)\end{array}$ & $\begin{array}{c}55(100.0) \\
0(0)\end{array}$ \\
\hline $\begin{array}{l}\text { How has perception of other pro- } \\
\text { fessions changed }\end{array}$ & $\begin{array}{l}\text { Better } \\
\text { Worse }\end{array}$ & $\begin{array}{c}16(94.1) \\
1(5.9)\end{array}$ & $\begin{array}{c}19(100.0) \\
0(0)\end{array}$ & $\begin{array}{c}15(93.8) \\
1(6.3)\end{array}$ & $\begin{array}{c}50(96.2) \\
2(3.9)\end{array}$ \\
\hline
\end{tabular}

Vol. 6.1

April 2016

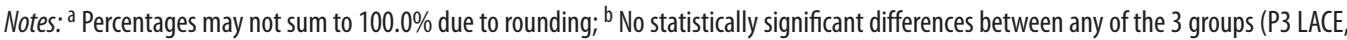
P4 IPE, or P3 LACE and P4 IPE) using either (hi-square tests or Fisher's exact test, as appropriate. 
JRIPE

7

Changes in

Pharmacy Students After Experiencing Interprofessional Education Activities

Tolleson, Ismail, Gill, Dello Stritto, Hatfield, Teal, Nelson, \& Hatfield

The results of the comparison for the four sliding scale questions among only IPE students on a pre-IPE versus post-IPE basis are found in Table 4. The levels increased on a pre- to post-IPE basis for each of the following questions: level of comfort with questioning a member of another profession, level of comfort with a member of another profession questioning the student, and level of reliability or accuracy of information from a nurse or a nursing student.

\section{Table 4. Comparison between students before and after interprofessional education (IPE) activities $(n=85)$}

\begin{tabular}{|l|l|c|c|c|}
\hline Item & & & \\
& Category & $n$ & Mean \pm SD & $p^{\text {a }}$ \\
\hline $\begin{array}{l}\text { Level of comfort with questioning a member of } \\
\text { another profession }\end{array}$ & Pre IPE & 85 & $\begin{array}{l}5.45 \pm 2.24 \\
7.22 \pm 1.62\end{array}$ & $<0.01$ \\
\hline Post IPE & 85 & Level of comfort with a member of another profession \\
questioning the student
\end{tabular}

Notes: ${ }^{\text {P Paired } t \text {-test; }}{ }^{\mathrm{b}}$ Scale: $0=$ least comfortable to $10=$ most comfortable; ${ }^{\mathrm{c}}$ Scale: $0=$ least reliable or accurate to $10=$ most reliable or accurate

The results of the comparison for the four sliding scale questions among only IPE students on a pre-IPE versus post-IPE basis, with each of the three IPE groups analyzed separately (P3 as LACE, P4 as IPE, and P3 as LACE and P4 as IPE combined) are found in Table 5. For the two questions: level of comfort with questioning a member of another profession and level of comfort with a member of another profession questioning the student; the levels increased on a pre-IPE to post-IPE basis for each of three groups (P3 LACE, P4 IPE, and P3 LACE and P4 IPE combined). The students who experienced only P3 LACE showed an increase from pre-IPE to post-IPE regarding their perception of the level of reliability or accuracy of information from a nurse or nursing student.

No statistically significant results were found in the perceived level of comfort and level of reliability between students with no IPE experience (using a traditional pre-test) and students with IPE experience(s) (using a post-test).

Journal of Research in Interprofessional Practice and Education

Vol. 6.1

April 2016

Results from the response shift analysis are found in Table 6. For the two questions: level of comfort with questioning a member of another profession and level of comfort with a member of another profession questioning the student: the baseline levels were higher for students with no IPE (using a traditional pre-test) than for students with IPE (using a retrospective pre-test). 
JRIPE

8

Changes in

Pharmacy Students After Experiencing Interprofessional Education Activities

Tolleson, Ismail, Gill, Dello Stritto, Hatfield, Teal, Nelson, \& Hatfield
Journal of Research in Interprofessional Practice and Education

Vol. 6.1

April 2016
Journal of Research in Interprofessional Practice and Education

Table 5. Comparison between students before and after interprofessional education (IPE) activities, by IPE level

\begin{tabular}{|c|c|c|c|c|}
\hline Item & Category & $n$ & Mean $\pm S D$ & $p^{\mathrm{a}}$ \\
\hline \multicolumn{5}{|c|}{ P3 LACE Students } \\
\hline $\begin{array}{l}\text { Level of comfort with questioning a member of } \\
\text { another profession }{ }^{b}\end{array}$ & $\begin{array}{l}\text { Pre IPE } \\
\text { Post IPE }\end{array}$ & $\begin{array}{l}24 \\
24\end{array}$ & $\begin{array}{l}4.83 \pm 2.62 \\
7.33 \pm 1.71\end{array}$ & $<0.01$ \\
\hline $\begin{array}{l}\text { Level of comfort with a member of another profession } \\
\text { questioning the student }{ }^{b}\end{array}$ & $\begin{array}{l}\text { Pre IPE } \\
\text { Post IPE }\end{array}$ & $\begin{array}{l}24 \\
24\end{array}$ & $\begin{array}{l}4.54 \pm 2.73 \\
6.92 \pm 1.95\end{array}$ & $<0.01$ \\
\hline $\begin{array}{l}\text { Level of reliability or accuracy of information from a } \\
\text { nurse or a nursing student }{ }^{c}\end{array}$ & $\begin{array}{l}\text { Pre IPE } \\
\text { Post IPE }\end{array}$ & $\begin{array}{l}24 \\
24\end{array}$ & $\begin{array}{l}5.58 \pm 2.45 \\
6.75 \pm 1.73\end{array}$ & 0.02 \\
\hline $\begin{array}{l}\text { Level of reliability or accuracy of information from a } \\
\text { physician or a medical student }{ }^{c}\end{array}$ & $\begin{array}{l}\text { Pre IPE } \\
\text { Post IPE }\end{array}$ & $\begin{array}{l}24 \\
24\end{array}$ & $\begin{array}{l}7.38 \pm 1.81 \\
7.71 \pm 1.43\end{array}$ & 0.28 \\
\hline \multicolumn{5}{|c|}{ P4 IPE Students } \\
\hline $\begin{array}{l}\text { Level of comfort with questioning a member of } \\
\text { another profession }{ }^{b}\end{array}$ & $\begin{array}{l}\text { Pre IPE } \\
\text { Post IPE }\end{array}$ & $\begin{array}{l}32 \\
32\end{array}$ & $\begin{array}{l}6.09 \pm 1.96 \\
7.38 \pm 1.26\end{array}$ & $<0.01$ \\
\hline $\begin{array}{l}\text { Level of comfort with a member of another profession } \\
\text { questioning the student }{ }^{b}\end{array}$ & $\begin{array}{l}\text { Pre IPE } \\
\text { Post IPE }\end{array}$ & $\begin{array}{l}32 \\
32\end{array}$ & $\begin{array}{l}5.81 \pm 1.93 \\
7.09 \pm 1.35\end{array}$ & $<0.01$ \\
\hline $\begin{array}{l}\text { Level of reliability or accuracy of information from a } \\
\text { nurse or a nursing student }{ }^{c}\end{array}$ & $\begin{array}{l}\text { Pre IPE } \\
\text { Post IPE }\end{array}$ & $\begin{array}{l}32 \\
32\end{array}$ & $\begin{array}{l}5.81 \pm 1.55 \\
6.19 \pm 1.57\end{array}$ & 0.06 \\
\hline $\begin{array}{l}\text { Level of reliability or accuracy of information from a } \\
\text { physician or a medical student }{ }^{c}\end{array}$ & $\begin{array}{l}\text { Pre IPE } \\
\text { Post IPE }\end{array}$ & $\begin{array}{l}32 \\
32\end{array}$ & $\begin{array}{l}6.97 \pm 1.18 \\
6.97 \pm 1.31\end{array}$ & 1.00 \\
\hline \multicolumn{5}{|c|}{ P3 LACE and P4 IPE Students } \\
\hline $\begin{array}{l}\text { Level of comfort with questioning a member of } \\
\text { another profession }{ }^{b}\end{array}$ & $\begin{array}{l}\text { Pre IPE } \\
\text { Post IPE }\end{array}$ & $\begin{array}{l}29 \\
29\end{array}$ & $\begin{array}{l}5.24 \pm 2.10 \\
6.97 \pm 1.90\end{array}$ & $<0.01$ \\
\hline $\begin{array}{l}\text { Level of comfort with a member of another profession } \\
\text { questioning the student }{ }^{b}\end{array}$ & $\begin{array}{l}\text { Pre IPE } \\
\text { Post IPE }\end{array}$ & $\begin{array}{l}29 \\
29\end{array}$ & $\begin{array}{l}5.62 \pm 1.99 \\
7.07 \pm 1.81\end{array}$ & $<0.01$ \\
\hline $\begin{array}{l}\text { Level of reliability or accuracy of information from a } \\
\text { nurse or a nursing student }{ }^{c}\end{array}$ & $\begin{array}{l}\text { Pre IPE } \\
\text { Post IPE }\end{array}$ & $\begin{array}{l}29 \\
29\end{array}$ & $\begin{array}{l}6.28 \pm 1.44 \\
6.38 \pm 1.59\end{array}$ & 0.72 \\
\hline $\begin{array}{l}\text { Level of reliability or accuracy of information from a } \\
\text { physician or a medical student }{ }^{c}\end{array}$ & $\begin{array}{l}\text { Pre IPE } \\
\text { Post IPE }\end{array}$ & $\begin{array}{l}29 \\
29\end{array}$ & $\begin{array}{l}7.00 \pm 1.49 \\
6.93 \pm 1.60\end{array}$ & 0.81 \\
\hline
\end{tabular}

Notes: ${ }^{\mathrm{a}}$ Paired t-test; ${ }^{\mathrm{b}}$ Scale: $0=$ =east comfortable to $10=$ most comfortable; ${ }^{\mathrm{C}}$ Scale: $0=$ least reliable or accurate to $10=$ most reliable or accurate 
JRIPE

9

Changes in

Pharmacy Students

After Experiencing

Interprofessional

Education Activities

Tolleson, Ismail, Gill, Dello Stritto, Hatfield, Teal, Nelson, \& Hatfield
Journal of Research in Interprofessional Practice and Education

Vol. 6.1

April 2016
Journal of Research in Interprofessional Practice and Education

Table 6. Comparison between students with no interprofessional education (IPE) (traditional pretest) and students with IPE (retrospective pretest) $(n=85)$

\begin{tabular}{|l|c|c|c|c|}
\hline \multicolumn{1}{|c|}{ Item $^{\mathrm{a}}$} & Category $^{\mathrm{a}}$ & $n$ & Mean \pm SD & $p^{\mathrm{b}}$ \\
\hline $\begin{array}{l}\text { Level of comfort with questioning a member of } \\
\text { another professionc }\end{array}$ & $\begin{array}{c}\text { Trad Pre } \\
\text { Retro Pre }\end{array}$ & $\begin{array}{c}101 \\
85\end{array}$ & $\begin{array}{c}7.00 \pm 2.06 \\
5.45 \pm 2.24\end{array}$ & $<0.01$ \\
\hline $\begin{array}{l}\text { Level of comfort with a member of another profession } \\
\text { questioning the student }^{c}\end{array}$ & $\begin{array}{c}\text { Trad Pre } \\
\text { Retro Pre }\end{array}$ & $\begin{array}{c}101 \\
85\end{array}$ & $\begin{array}{c}6.61 \pm 2.20 \\
5.39 \pm 2.24\end{array}$ & $<0.01$ \\
\hline $\begin{array}{l}\text { Level of reliability or accuracy of information from a } \\
\text { nurse or a nursing student }{ }^{\mathrm{d}}\end{array}$ & $\begin{array}{c}\text { Trad Pre } \\
\text { Retro Pre }\end{array}$ & $\begin{array}{c}101 \\
85\end{array}$ & $\begin{array}{c}6.07 \pm 1.86 \\
5.91 \pm 1.82\end{array}$ & 0.55 \\
\hline $\begin{array}{l}\text { Level of reliability or accuracy of information from a } \\
\text { physician or a medical student }\end{array}$ & $\begin{array}{c}\text { Trad Pre } \\
\text { Retro Pre }\end{array}$ & $\begin{array}{c}101 \\
85\end{array}$ & $\begin{array}{c}7.30 \pm 1.51 \\
7.09 \pm 1.48\end{array}$ & 0.36 \\
\hline
\end{tabular}

Notes: ${ }^{\mathrm{a}}$ Trad Pre $=$ Traditional Pretest. Retro Pre $=$ Retrospective Pretest $;{ }^{\mathrm{b}}$ Unpaired $\mathrm{t}$-test; ${ }^{\mathrm{c}} \mathrm{Scale}: 0=$ least comfortable to $10=$ most comfortable; ${ }^{\mathrm{d}}$ Scale: $0=$ least reliable or accurate to $10=$ most reliable or accurate

\section{Discussion}

This study assessed the impact of IPE experiences on pharmacy students' perceived level of comfort and level of reliability regarding other healthcare professionals. Significant results were revealed regarding changes in self-reported perceptions on a pre- versus post-IPE basis. This could be a reflection of perceived change, rather than an actual change in attitude.

No significantly different results were found when comparing the perceptions of students who have not experienced IPE (using a traditional pre-test) with perceptions of students who have experienced IPE (using a post-test), possibly due to a response shift. Students with no IPE do not have the experience to gauge or calibrate an informed perception versus students who have experienced IPE. We assume that the cohort of students with no IPE experience were comparable to the cohort of IPE students prior to their IPE experience(s). The demographic and descriptive characteristics of both cohorts help to support this assumption. We propose that the IPE students were able to more accurately calibrate their pre-test and post-test perceptions through a response shift.

A retrospective pre-test was used in lieu of a traditional pre-test to minimize the effect of response shift bias among students with any IPE experience. Retrospective pre-tests have been studied extensively and have been shown to be effective in cases where a shift in the responder's basis is possible [15]. Kim Nimon and Jeff Allen performed an extensive review of the retrospective pre-test, finding that it has relevant application when applied properly [13-15]. Theodore A. Lamb and Raymond Tschillard and Jennifer Martineau and Kelly Hannum argued that retrospective pretests should be used instead of traditional pre-tests [16,17]. The results highlighted the potential for bias regarding certain items with the use of a traditional pre-test. This finding is corroborated by the study by Scott Bolesta and Joyce V. Chmil using 
10

Changes in

Pharmacy Students After Experiencing Interprofessional Education Activities

Tolleson, Ismail, Gill, Dello Stritto, Hatfield, Teal, Nelson, \& Hatfield

Journal of Research in Interprofessional Practice and Education

Vol. 6.1

April 2016 a similar methodology (retrospective pre-test and traditional pre-test) among a similar cohort of pharmacy students experiencing IPE [18].

When examining pre- and post-IPE, survey responses showed that students who have completed either or both IPE activities were significantly more comfortable with questioning other professions and being questioned by other professions. This is possibly due to the students seeing the other professions on a student-to-student level and being able to relate to the other disciplines through their common experiences. These changes in perception could translate to improved interprofessional communication as pharmacy students recognize that being questioned should not be taken personally, and questioning others should be thought of as a means to improve patient care. The analysis also shows that students who have had IPE experience feel that nursing students and nurses provide significantly more reliable and accurate information than they thought before IPE. The results for the perceived reliability and accuracy of information from medical students and physicians also reveal an increase after IPE, but this was not significant. One explanation for the difference in significance of reliability or accuracy of information from the nursing profession versus the medical profession could be that fewer of the pharmacy students reported having previous working experience with nurses and nursing students $(n=72)$ than physicians and medical students $(n=123)$. Another explanation is that pharmacy students learned more about the nursing profession and found that nurses can provide reliable and accurate information. Regarding perceptions toward medical students, the pharmacy students may still believe accurate and reliable information is provided, but they may be more cautious with that information because they now know physicians are capable of human error. It is also possible that prior to IPE, pharmacy students were already more likely to question the reliability and accuracy of information from a physician, and this remained the same after the IPE. Regardless of the reason, this finding reinforces a critical lesson in patient care: that when in doubt, one should not be reserved in questioning another healthcare professional.

Students' responses to the four sliding scale questions after IPE experience (using a retrospective pre-test) were not significantly different than students who have not experienced IPE (using a traditional pre-test). However, in all four sliding scale questions, students who have experienced IPE consistently scored their pre-IPE answers lower than students who have not experienced IPE. We propose the explanation for this is the ability for students who have had an IPE experience to recalibrate their responses based on their IPE experience, while students who have not had such an experience cannot accurately assess their baseline. The difference is the response shift.

Similar results were revealed upon further analysis of the subgroups of students with IPE experience. All subgroups (students who only experienced the LACE class as a P3, students who only experienced the IPE activity as P4s, and students who experienced both) were more comfortable with both questioning other professionals and being questioned by other professionals. This perceived change demonstrates an increased willingness to communicate with other professionals about possible problems related to patient care. While working with the other professions, the students likely realized that everyone was working toward the common goal of improving 
11

Changes in

Pharmacy Students After Experiencing Interprofessional Education Activities

Tolleson, Ismail, Gill, Dello Stritto, Hatfield, Teal, Nelson, \& Hatfield

Journal of Research in Interprofessional Practice and Education

Vol. 6.1

April 2016 patient outcomes. To reach their goal, the students had to work together as a healthcare team, instead of as separate entities. The students were able to see that being questioned by other professions or questioning other professions is not meant to be personal, but rather is meant to clarify or ensure that correct decisions have been made for the patient, thus improving patient care. Students who have only experienced the LACE NPLH visit also demonstrated a significant increase in their feeling of reliability and accuracy of information from the nursing profession and a non-significant increase in their feeling of reliability and accuracy of information from the medical profession. Responses from students who participated in only the IPE activity as a P4 showed an increase in the feeling of reliability and accuracy of information for the nursing profession, though it was not significant. In the same group, the level of reliably accurate information from the medical profession remained relatively unchanged. Analysis of the responses from students who experienced both of the activities showed a non-significant increase in the feeling of reliability and accuracy of information from the nursing profession and a non-significant decrease in the feeling of reliability and accuracy of information from the medical profession. The differences seen in these groups could be from different levels of experience with and exposure to the other professions. Fourth-year pharmacy students frequently work with medical students, physicians, and nurses while on their Advanced Pharmacy Practice Experience (APPE) rotations. Some students commented that past work-related or APPE experiences outweighed their IPE experiences in regards to their perceptions of the other professions.

Almost all (94.3\%) of the IPE-experienced students felt their IPE was beneficial, and 80.5 percent of these students indicated that IPE affected how they work with other professions. To gain more insight into potential perceived changes, students were asked to explain how or why working with different professions has been affected. Many responses included more insight into the other professions and the knowledge they hold, along with feeling more like a team and being more comfortable with communicating concerns. This is reflected in the responses to the questions regarding the comfort level of questioning other professions or being questioned by other professionals. Most students (77.7\%) were more willing to ask for assistance, clarification, or the services of the other professions. This further supports that IPE during the education of healthcare professionals is beneficial to healthcare teams through improvements in communications between professions.

There were some limitations to our study. Only pharmacy students were surveyed. Also, the IPE situations faced by the students in the LACE class had a high degree of variability due to the nature of home visits, while the P4 IPE activity was very similar for each student. Another limitation of our study is that it only accounted for two IPE experiences at UHCOP, and no other experiences were considered. Many of the students responded that they have worked with physicians and nurses prior to their IPE experience at UHCOP, and these experiences may have impacted their IPE activity. Lastly, the instrument had not been previously validated.

This study revealed that pharmacy students who have experienced any form of IPE feel more comfortable with questioning other healthcare professionals than stu- 
12

Changes in Pharmacy Students After Experiencing Interprofessional Education Activities

Tolleson, Ismail, Gill, Dello Stritto, Hatfield, Teal, Nelson, \& Hatfield

Journal of Research in Interprofessional Practice and Education

Vol. 6.1

April 2016 dents with no IPE. Improved levels of communication among healthcare professionals through questioning could improve patient outcomes, underscoring the benefits of incorporating IPE into healthcare education. Additional research is needed to more clearly define the benefits and design of IPEs.

\section{Abbreviations}

$\begin{array}{ll}\text { AACP } & \text { American Association of Colleges of Pharmacy } \\ \text { BCM } & \text { Baylor College of Medicine } \\ \text { IPE } & \text { Interprofessional Education } \\ \text { IPEC } & \text { Interprofessional Education Collaborative } \\ \text { IPPE } & \text { Introductory Pharmacy Practice Experience } \\ \text { LACE } & \text { Longitudinal Ambulatory Clinical Experience } \\ \text { NPLH } & \text { No Place Like Home } \\ \text { UHCOP } & \text { University of Houston College of Pharmacy }\end{array}$

\section{References}

1. Buring, Shauna M., Bhushan, Alok, Broeseker, Amy, Conway, Susan, Duncan-Hewitt, Wendy, Hansen, Laura, \& Westberg, Sarah. (2009). Interprofessional education: Definitions, student competencies, and guidelines for implementation. American Journal of Pharmaceutical Education, 73, 1-8.

2. Centre For The Advancement Of Interprofessional Education. (2002). Defining IPE. URL: http://caipe.org.uk/about-us/defining-ipe/ [July 26, 2015].

3. Bridges, Diane R., Davidson, Richard A., Odegard Peggy S., Maki Ian V., \& Tomkowiak John (2011). Interprofessional collaboration: Three best practice models of interprofessional education. Medical Education Online, 16, 1-21.

4. Baker, David P., Gustafson, Sigrid, Beaubien, Jeff, Salas, Eduardo, \& Barach, Paul. (2005). Medical teamwork and patient safety: The evidence-based relation. Report prepared for the Center for Quality Improvement \& Patient Safety, Agency for Healthcare Research \& Quality (AHRQ).

5. Burton, Andrea, Hammick, Marilyn, \& Hoffman, Steven J. (2010). Framework for action on interprofessional education and collaborative practice. Geneva, Switzerland: Health Professions Network Nursing and Midwifery Office within the Department of Human Resources for Health, World Health Organization.

6. Accreditation Council for Pharmacy Education. (2011). Accreditation standards and guidelines for the professional program in pharmacy leading to the doctor of pharmacy degree. URL: www.acpeaccredit.org/pdf/FinalS2007Guidelines2.0.pdf [July 26, 2015].

7. Jones, Kim M., Blumenthal, Donald K., Burke, John M., Condren, Michelle, Hansen, Richard, Holiday-Goodman, Monica, \& Peterson, Charles D. (2012). Interprofessional education in introductory pharmacy practice experiences at US colleges and schools of pharmacy. American Journal of Pharmaceutical Education, 76, 1-15.

8. Remington, Tami L., Foulk, Mariko A., \& Williams, Brent C. (2006). Evaluation of evidence for interprofessional education. American Journal of Pharmaceutical Education, 70, 1-14.

9. Zorek, Joseph A., MacLaughlin, Eric J., Fike, David S., MacLaughlin, Anitra A., Samiuddin, Mohammed, \& Young, Rodney B. (2014). Measuring changes in perception using Student Perceptions of Physician-Pharmacist Interprofessional Clinical Education (SPICE) instrument. BMC Medical Education, 14, 1-7.

10. Michalec, Barret, Giordano, Carolyn, Arenson, Christine, Antony, Reena, \& Rose, Molly (2013). Dissecting first-year students' perceptions of health profession groups: Potential barriers to interprofessional education. Journal of Allied Health, 42, 202-213.

11. Bolesta, Scott, \& Chmil, Joyce V. (2014). Interprofessional education among student health professionals using human patient simulation. American Journal of Pharmaceutical Education, 75, 1-9.

12. Baylor College of Medicine. (2015). Baylor College of Medicine student handbook: Longitudinal ambulatory clinical experience. Houston, TX: Baylor College of Medicine. 


\section{JRIPE}

Changes in

Pharmacy Students After Experiencing Interprofessional Education Activities

Tolleson, Ismail, Gill, Dello Stritto, Hatfield, Teal, Nelson, \& Hatfield

\section{Journal of Research in Interprofessional Practice and Education}

13. Howard, George S., Ralph, Kenneth M., Gulanick, Nancy A., Maxwell, Scott E., Nance, Don W., \& Gerber, Sterling K. (1979). Internal invalidity in pretest-posttest self-report evaluations and a reevaluation of retrospective pretests. Applied Psychological Measurement, 3, 1-23.

14. Howard, George S. (1980). Response-shift bias a problem in evaluating interventions with pre/post self-reports. Evaluation Review, 4, 93-106.

15. Nimon, Kim, \& Allen, Jeff. (2007). A review of the retrospective pretest: Implications for performance improvement evaluation and research. Workforce Education Forum, 44, 36-55.

16. Lamb, Theodore A., \& Tschillard, Raymond. (2005). Evaluating learning in professional development workshops: Using the retrospective pretest. Journal of Research in Professional Learning, 1, 1-9.

17. Martineau, Jennifer, \& Hannum, Kelly (2004). Evaluating the impact of leadership development: A professional guide. Greensboro, NC: Center for Creative Leadership.

18. Bolesta, Scott, \& Chmil, Joyce V. (2014). Interprofessional education among student health professionals using human patient simulation. American Journal of Pharmaceutical Education, 78, 1-9. 


\section{JRIPE}

Changes in Pharmacy Students After Experiencing Interprofessional Education Activities

Tolleson, Ismail, Gill, Dello Stritto, Hatfield, Teal, Nelson, \& Hatfield
Journal of Research in Interprofessional Practice and Education

\section{Appendix}

\section{Questionnaire for Pharmacy Students}

Q1 Please answer the following questions honestly. This survey is anonymous.

Q2 What is your gender?

Male (1)

Female (2)

Q3 What year are you?

P3 (1)

$\mathrm{P} 4(2)$

Q4 What is your age?

$1(1)$

Q5 Have you previously worked with nurses or nursing students? (excluding during the LACE Project and the Interprofessional Education activity for APPEs)

Yes (1)

No (2)

Q6 Do you know anyone on a personal basis that is a nurse or in nursing school? Yes (1)

No (2)

Q7 Overall, how would you rate your attitude toward the nursing profession? Positive (1)

Neutral (2)

Negative (3)

Q8 Have you previously worked with physicians or medical students? (excluding during the LACE Project and the Interprofessional Education activity for APPEs)

Yes (1)

No (2)

Q9 Do you know anyone on a personal basis that is a physician or in medical school?

Yes (1)

No (2) 


\section{JRIPE}

15

Changes in

Pharmacy Students After Experiencing Interprofessional Education Activities

Tolleson, Ismail, Gill, Dello Stritto, Hatfield, Teal, Nelson, \& Hatfield
Journal of Research in Interprofessional Practice and Education

Q10 Overall, how would you rate your attitude toward the medical profession? Positive (1)

Neutral (2)

Negative (3)

Q11 Have you participated in any Interprofessional Education (IPE) activities? (Ex. LACE Project or IPE for APPE's)

Yes, only LACE as a P3 (1)

Yes, LACE as a P3 AND IPE as a P4 (2)

Yes, only IPE as a P4 (3)

No (4)

Q12 Overall, do you feel that the Interprofessional Activity(ies) you participated in was/were beneficial?

Yes (1)

No (2)

Q13 Have you previously had a positive experience with physicians or medical students?

Yes (1)

No (2)

Q14 Have you previously had a negative experience with physicians or medical students

Yes (1)

No (2)

Q15 Have you previously had a positive experience with nurses or nursing students?

Yes (1)

No (2)

Q16 Have you previously had a negative experience with nurses or nursing students?

Yes (1)

No (2)

Q17 How comfortable were you BEFORE the IPE Activity with questioning a member of another profession, on a scale from $0-10$, with 0 being the least comfortable and 10 being the most comfortable?

$1(1)$
Journal of Research in Interprofessional Practice and Education

Vol. 6.1

April 2016 


\section{JRIPE}

16

Changes in

Pharmacy Students After Experiencing Interprofessional

Education Activities

Tolleson, Ismail, Gill, Dello Stritto, Hatfield, Teal, Nelson, \& Hatfield
Journal of Research in Interprofessional Practice and Education

Q18 How comfortable were you AFTER the IPE Activity with questioning a member of another profession, on a scale from $0-10$, with 0 being the least comfortable and 10 being the most comfortable? $1(1)$

Q19 How comfortable were you BEFORE the IPE Activity with a member of another profession questioning you, on a scale from $0-10$, with 0 being the least comfortable and 10 being the most comfortable? $1(1)$

Q20 How comfortable were you AFTER the IPE Activity with a member of another profession questioning you, on a scale from $0-10$, with 0 being the least comfortable and 10 being the most comfortable? Click to write Choice 1 (1)

Q21 How reliable/accurate did you believe information from a physician or medical student to be BEFORE the IPE Activity, on a scale from 0-10, with 0 being the least reliable/accurate and 10 being the most reliable/accurate? Click to write Choice 1 (1)

Q22 How reliable/accurate did you believe information from a physician or medical student to be AFTER the IPE Activity, on a scale from 0-10, with 0 being the least reliable/accurate and 10 being the most reliable/accurate? Click to write Choice 1 (1)

Q23 How reliable/accurate did you believe information from a nurse or nursing student to be BEFORE the IPE Activity, on a scale from $0-10$, with 0 being the least reliable/accurate and 10 being the most reliable/accurate? Click to write Choice 1 (1)

Q24 How reliable/accurate did you believe information from a nurse or nursing student to be AFTER the IPE Activity, on a scale from 0-10, with 0 being the least reliable/accurate and 10 being the most reliable/accurate?

Click to write Choice 1 (1)

Q25 Do you believe that the IPE Activity has affected how you work and will continue to work with members of these professions?

Yes (1)

No (2)

Neutral (3) 


\section{JRIPE}

17

Changes in

Pharmacy Students After Experiencing Interprofessional Education Activities

Tolleson, Ismail, Gill, Dello Stritto, Hatfield, Teal, Nelson, \& Hatfield

Journal of Research in Interprofessional Practice and Education

Q26 Has your perception of the professions you worked with changed due to your experience during the IPE Activity

Yes (1)

No (2)

Q27 Do you feel that, after your experience, you are more willing to ask for assistance, clarification, or services from physicians or nurses?

Yes (1)

No (2)

Neutral (3)

Answer If Do you believe that the IPE Activity has affected how you ... Yes Is Selected

Q28 How has Interprofessional Education affected how you work with members of the nursing and medical professions?

Positively (1)

Negatively (2)

Answer If Do you believe that the IPE Activity has affected how you ... Yes Is Selected

Q29 Please explain your previous answer.

Answer If Do you believe that the IPE Activity has affected how you ... No Is Selected

Q30 Why do you think you were not affected by your Interprofessional Education experience?

Answer If Has your perception of the professions you worked with ch ... Yes Is Selected

Q31 How has your perception of the professions changed?

Better (1)

Worse (2)

Answer If Has your perception of the professions you worked with ch ... Yes Is Selected

Journal of Research in Interprofessional Practice and Education
Q32 Please explain how your perception has changed. 


\section{JRIPE}

18

Changes in

Pharmacy Students After Experiencing Interprofessional Education Activities

Tolleson, Ismail, Gill, Dello Stritto, Hatfield, Teal, Nelson, \& Hatfield
Journal of Research in Interprofessional Practice and Education

Q33 Overall, how would your rate your attitude toward the nursing profession? Positive (1)

Neutral (2)

Negative (3)

Q34 Overall, how would you rate your attitude toward the medical profession? Positive (1)

Neutral (2)

Negative (3)

Q35 How comfortable are you with questioning a member of another profession, on a scale from $0-10$, with 0 being the least comfortable and 10 being the most comfortable?

Click to write Choice 1 (1)

Q36 How comfortable are you with a member of another profession questioning you, on a scale from $0-10$, with 0 being the least comfortable and 10 being the most comfortable?

$1(1)$

Q37 How reliable/accurate do you believe information from a physician or medical student to be, on a scale from $0-10$, with 0 being the least reliable/accurate and 10 being the most reliable/accurate? $1(1)$

Q38 How reliable/accurate did you believe information from a nurse or nursing student to be, on a scale from $0-10$, with 0 being the least reliable/accurate and 10 being the most reliable/accurate? $1(1)$ 